\title{
Age-related hearing loss and speech perception disorder: the broken interface between healthcare professionals and older adults
}

\author{
Rodolfo Sardone $^{1} \cdot$ Madia Lozupone ${ }^{2} \cdot$ Francesco Panza $^{1}$ (I)
}

Published online: 12 August 2020

(c) European Geriatric Medicine Society 2020

Hearing status is vital not only for communication but also for health, and affects the quality of life. There is evidence that older people with age-related hearing loss (ARHL) are at greater risk for adverse health outcomes, such as cognitive impairment, increased social isolation, psychiatric disorders, and a greater need of support by others $[1,2]$. The recognition and treatment of ARHL should be the responsibility of all healthcare professionals working with older adults. The narrative review by Smith et al. [3] explored a topic usually kept in the background in the clinical and functional evaluation of older patients. These authors quantified the extent to which ARHL is accounted for in studies of older adult interactions with healthcare professionals. Their intent was to underline the impact of sensory interface status, especially the disorders related to the decline of hearing and speech comprehension functions occurring in older age, commonly defined as ARHL. Smith and colleagues' narrative review highlights that assessment at such a level of personal participation cannot exclude a check of the necessary integrity of communication channels, first of all those related to hearing disorders [3]. According to Smith and colleagues [3], despite the high prevalence of ARHL, across the health professions, very few studies on healthcare professional-older patient communications have incorporated hearing loss as a variable in their study design or analyses.

In fact, ARHL, or presbycusis, is the most frequent sensory impairment in people aged $65+$ years, with an estimated prevalence of $18 \%$ among older people in high-income

Web of Science Researcher ID: M-6804-2017.

Francesco Panza

f_panza@hotmail.com; geriat.dot@uniba.it

1 Frailty Phenotypes Research Unit, "Salus in Apulia Study", National Institute of Gastroenterology "Saverio de Bellis", Research Hospital, Castellana Grotte, Bari, Italy

2 Neurodegenerative Disease Unit, Department of Basic Medicine, Neuroscience, and Sense Organs, University of Bari Aldo Moro, Bari, Italy countries [4], and showing an exponential increase with aging, up to some degree of hearing loss in $100 \%$ of centenarians [5]. The two main ARHL features are peripheral ARHL and age-related central auditory processing disorders (CAPD). ARHL is not only determined by senescence of the inner ear cells, but also is a complex impairment that mostly involves the central auditory pathways [6]. Peripheral ARHL is largely related to a progressive decline of cochlear function and is assessed by pure tone audiometry. The term agerelated CAPD refers to central auditory pathway disorders, assessed only in peripheral normal-hearing subjects who have difficulties in understanding speech against background noise or competitive speech [6]. The sensorial impairment deriving from ARHL, considered as a frailty status, causes substantial interference with the communication channels. In their article [3], Smith and colleagues highlighted the importance of the integrity of the communication channel, taking into account hearing functions. The consequences of ARHL in daily life include difficulty in interpreting speech sounds, which often result in a reduced ability to communicate, impaired physical and social functions, and a diminished quality of life [7].

Several emerging findings demonstrate that hearing deficits are associated with different phenotypes of frailty [8-10], defined as an intermediate functional status of the aging process associated with multiple adverse healthrelated outcomes (i.e., falls, mobility loss, disability, and early mortality). Among these phenotypes, sensorial frailty appears to be strictly linked to ARHL features [8]. In fact, frailty is acknowledged to be not only a biological or physiological state, but also a multi-dimensional concept that includes physical, cognitive, sensorial, nutritional, psychological/depressive, and sensorial phenotypes. Different models of frailty (physical/biological model, deficit accumulation model, and bio-psychosocial/multi-dimensional model), also act as possible modulators of the sensorial changes-cognition link in older age [8]. Understanding the relationship between the different features of ARHL 
and frailty phenotypes, particularly physical and cognitive frailty, may be very important because correct ARHL management may be an effective strategy to prevent frailty or at least moderate its clinical evolution and consequences. Frailty is a product of a thorough, close evaluation of the patient and cannot be properly evaluated in subjects with an impaired communication channel. Therefore, it seems hard to disentangle whether the effect of ARHL on frailty is actually a biological problem or a difficulty related to the inability to understand the clinical interview. The withdrawal of older adults from situations in which they may have difficulty in hearing and communicating may explain behavioral mechanisms which could contribute to the development of social isolation, loneliness and consequent cognitive decline and depression [11]. Psychiatrists are also in an excellent position to identify ARHL given that when they administer a mental status examination, the older adult may reveal the disorder through answers that suggest that the question was misunderstood [12]. Furthermore, ARHL is associated with many psychiatric disorders, including depression, anxiety, and psychoses.

A multipronged approach is needed to improve the accessibility and affordability of hearing healthcare. A modern comprehensive geriatric assessment (CGA) is not merely a functional physical examination, but a process which is used to manage frail or vulnerable older people. In fact, CGA is a multidisciplinary diagnostic and treatment process that identifies medical, psychosocial, and functional capabilities of older adults to develop a coordinated plan to maximize overall health during aging [13]. It is interdisciplinary (physicians but also nurses and allied healthcare professionals), multi-dimensional (medical diagnoses, functional impairments, environmental, and social issues), developing a personalized health pattern with personalized interventions. The effectiveness of CGA programs may be modified by particular settings or specific clinical conditions, such as cognitive impairment or ARHL [13]. Furthermore, hearing impairment could also suggest a poor compliance with the prescriptions made by healthcare professionals, thus causing possible damage due to failure to adhere to drug therapies. This is indirectly supported by some evidence that showed how ARHL could increase mortality in older adults [14], along with severe health-related adverse outcomes, such as cardiovascular diseases [15, 16] and falls [17].

Another point raised in the narrative review by Smith et al. [3] is that the understanding of informed consent could be affected by poor hearing function. This is not a specific clinical problem, but it poses a huge ethical problem, which should be investigated before or during the making of important patient choices. Informed consent is an essential element in the doctor-patient relationship. In particular, obtaining valid informed consent from patients with neurodegenerative diseases with a cognitive focus is currently a critical issue [18]. Particularly, the decision-making capacity seems to be strongly affected by the cognitive status in older patients [19]. This point is very important, since ARHL is one of the most important modifiable risk factors for the development of cognitive impairment in late life [20-23], especially in its central component, i.e., age-related CAPD. In particular, this disorder seems to be in itself a neurodegenerative process of the central auditory pathways [23]. The evidence is so strong that this age-related CAPD-cognition link has been described using the provocative term "the cognitive ear," suggesting that hearing functions are not only processed by the ear and by the auditory cortex, but also by other associative cortical areas [6].

In the last two decades, the important role of ARHL in the development of dementia and late-life cognitive decline has emerged from several population-based studies [22]. In particular, age-related CAPD was suggested as a strong risk factor for the development of Alzheimer's disease [21], and more recently, this association was extended also to the prodromal forms of dementia, such as mild cognitive impairment [23]. For this reason, before any important choice is submitted to the patient, we are convinced that the presence of associated auditory and cognitive deficits should be evaluated, even retrospectively.

In conclusion, we strongly agree with one of the principal conclusions of the narrative review by Smith et al. [3], namely that there is a notable lack of research, particularly focused on interventions designed to address the effects of ARHL on communication in clinical settings. Patients with ARHL and their caregivers similarly referred a significant negative impact on the social dimension of the quality of life [24], suggesting the importance of the early identification of hearing loss and offering rehabilitative support [25]. ARHL can represent a transverse confounding factor, both for the effectiveness of the CGA and for measuring the efficacy of therapeutic interventions. An effective CGA should routinely take into account the presence of ARHL, both central and peripheral. More extensively, every diagnostic process in subjects aged 65 years and older should also envisage the presence of health professionals, such as audiologists and speech therapists.

Acknowledgements The authors thank the "Salus in Apulia" Research Team. This manuscript is the result of the research work on frailty undertaken by the "Research Network on Aging" team, supported by the resources of the Italian Ministry of Health-Research Networks of National Health Institutes. The authors thank M.V. Pragnell, B.A., for her precious help as native English supervisor.

Author contributions RS, ML, and FP wrote the manuscript and did literature review. All co-authors read and approved the final version of the manuscript. 
Funding This study was funded by the Italian Ministry of Health with "Ricerca Corrente 2019" Grant.

\section{Compliance with ethical standards}

Conflict of interest On behalf of all authors, the corresponding author states that there is no conflict of interest.

Ethical approval This study does not contain any studies with human participants performed by any of the authors.

Informed consent For this type of study, formal consent is not required.

\section{References}

1. Lin FR, Yaffe K, Xia J et al (2013) Hearing loss and cognitive decline in older adults. JAMA Intern Med 173:293-299. https:// doi.org/10.1001/jamainternmed.2013.1868

2. Huang CQ, Dong BR, Lu ZC et al (2010) Chronic diseases and risk for depression in old age: a meta-analysis of published literature. Ageing Res Rev 9:131-141. https://doi.org/10.1016/j. arr.2009.05.005

3. Smith S, Nordin MAB, Hinchy T et al (2020) Impact of hearing loss on clinical interactions between older adults and health professionals: a systematic review. Eur Geriatr Med. https://doi. org/10.1007/s41999-020-00358-3

4. Olusanya BO, Neumann KJ, Saunders JE (2014) The global burden of disabling hearing impairment: a call to action. Bull World Health Organ 92:367-373. https://doi.org/10.2471/BLT.13.12872 8

5. Lin FR, Niparko JK, Ferrucci L (2011) Hearing loss prevalence in the United States. Arch Intern Med 171:1851-1852. https://doi. org/10.1001/archinternmed.2011.506

6. Sardone R, Battista P, Panza F et al (2019) The age-related central auditory processing disorder: silent impairment of the cognitive ear. Front Neurosci 13:327. https://doi.org/10.3389/fnins .2019.00619

7. Campbell VA, Crews JE, Moriarty DG et al (1999) Surveillance for sensory impairment, activity limitation, and health-related quality of life among older adults-United States, 1993-1997. MMWR CDC Surveill Summ 48:131-156

8. Panza F, Lozupone M, Sardone R et al (2019) Sensorial frailty: age-related hearing loss and the risk of cognitive impairment and dementia in later life. Ther Adv Chronic Dis 10:2040622318811000. https://doi.org/10.1177/2040622318 811000

9. Yoo M, Kim S, Kim BS et al (2019) Moderate hearing loss is related with social frailty in a community-dwelling older adults: the Korean Frailty and Aging Cohort Study (KFACS). Arch Gerontol Geriatr 83:126-130. https://doi.org/10.1016/j.archg er.2019.04.004

10. Kamil RJ, Betz J, Powers BB et al (2016) Association of hearing impairment with incident frailty and falls in older adults. J Aging Health 28:644-660. https://doi.org/10.1177/0898264315608730

11. Rutherford BR, Brewster K, Golub JS et al (2018) Sensation and psychiatry: Linking age-related hearing loss to late-life depression and cognitive decline. Am J Psychiatry 175:215-224. https://doi. org/10.1176/appi.ajp.2017.17040423
12. Blazer DG (2020) Hearing loss: the silent risk for psychiatric disorders in late life. Clin Geriatr Med 36:201-209. https://doi. org/10.1016/j.cger.2019.11.002

13. Pilotto A, Cella A, Pilotto A et al (2017) Three decades of Comprehensive Geriatric Assessment: evidence coming from different healthcare settings and specific clinical conditions. J Am Med Dir Assoc 18:192.e1-192.e11. https://doi.org/10.1016/j.jamda .2016.11.004

14. Genther DJ, Betz J, Pratt S et al (2015) Association of hearing impairment and mortality in older adults. J Gerontol A Biol Sci Med Sci 70:85-90. https://doi.org/10.1093/gerona/glu094

15. Fang Q, Wang Z, Zhan Y et al (2018) Hearing loss is associated with increased CHD risk and unfavorable CHD-related biomarkers in the Dongfeng-Tongji cohort. Atherosclerosis 271:70-76. https://doi.org/10.1016/j.atherosclerosis.2018.01.048

16. Fang Q, Lai X, Yang L, Wang Z, Zhan Y, Zhou L et al (2019) Hearing loss is associated with increased stroke risk in the Dongfeng-Tongji Cohort. Atherosclerosis 285:10-16. https://doi. org/10.1016/j.atherosclerosis.2019.03.012

17. Jiam NT-L, Li C, Agrawal Y (2016) Hearing loss and falls: a systematic review and meta-analysis. Laryngoscope 126:2587-2596. https://doi.org/10.1002/lary.25927

18. Carabellese F, Felthous AR, La Tegola D et al (2018) Qualitative analysis of the capacity to consent to treatment in patients with a chronic neurodegenerative disease: Alzheimer's disease/Analisi qualitativa sulla capacità a prestare consenso al trattamento in pazienti con malattie cronico degenerative neuropsicoorganiche: Demenza di Alzheimer. Int J Soc Psychiatry 64:26-36. https://doi. org/10.1177/0020764017739642

19. Han SD, Duke Han S, Boyle PA et al (2016) Discrepancies between cognition and decision making in older adults. Aging Clin Exp Res 28:99-108. https://doi.org/10.1007/s4052 0-015-0375-7

20. Lin FR, Ferrucci L, Metter EJ et al (2011) Hearing loss and cognition in the Baltimore Longitudinal Study of Aging. Neuropsychology 25:763-770. https://doi.org/10.1037/a0024238

21. Gates GA, Beiser A, Rees TS et al (2002) Central auditory dysfunction may precede the onset of clinical dementia in people with probable Alzheimer's disease. J Am Geriatr Soc 50:482-488. https://doi.org/10.1046/j.1532-5415.2002.50114.x

22. Loughrey DG, Kelly ME, Kelley GA et al (2018) Association of age-related hearing loss with cognitive function, cognitive impairment, and dementia. JAMA Otolaryngol-Head Neck Surg 144:115. https://doi.org/10.1001/jamaoto.2017.2513

23. Sardone R, Petronilla B, Donghia R et al (2020) Age-related central auditory processing disorder, MCI, and dementia in an older population of Southern Italy. J Otolaryngol Head Neck Surg. https ://doi.org/10.1177/0194599820913635

24. Lazzarotto S, Martin F, Saint-Laurent A et al (2019) Coping with age-related hearing loss: patient-caregiver dyad effects on quality of life. Health Qual Life Outcomes 17:86. https://doi.org/10.1186/ s12955-019-1161-6

25. Arlinger S (2003) Negative consequences of uncorrected hearing loss: a review. Int J Audiol 42(Suppl 2):2S17-2S20. https://doi. org/10.3109/14992020309074639

Publisher's Note Springer Nature remains neutral with regard to jurisdictional claims in published maps and institutional affiliations. 